\title{
Prospect Theory as Efficient Perceptual Distortion
}

\author{
By Michael WoodforD*
}

In rational choice theory, individuals are assumed always to choose the option that will provide them maximum utility. But actual choices must be based on subjective perceptions of the attributes of the available options, and the accuracy of these perceptions will always be limited by the information-processing capacity of one's nervous system.

In recent work (Woodford 2011), I propose a theory of valuation errors under the hypothesis that perceptions are as accurate as possible on average, given the statistical properties of the environment to which they are adapted, subject to a limit on processing capacity. The capacity limit requires that choice be based on a compressed representation of the choice situation; the data compression inevitably introduces distortions, but the resulting behavioral biases are actually efficient, when one takes account of the information-processing constraint. The theory is related to the "rational inattention" hypothesis of Christopher A. Sims (2011), but modified for closer conformity with psychophysical and neurobiological evidence regarding perceptual accuracy.

This hypothesis can explain a variety of anomalous aspects of economic choice in experimental settings. Here I discuss the explanation that it provides for choices over lotteries of the kind captured by the prospect theory of Daniel Kahneman and Amos Tversky (1979).

\section{Errors in Visual Perception}

To explain my hypothesis, it is useful to first consider the case of visual percep-

* Columbia University, 420 W. 118th St., New York, NY 10027 (email: mw2230@columbia.edu). Thanks to Dmitriy Sergeyev for research assistance; to Tom Cunningham, Daniel Kahneman, David Laibson, and Andrei Shleifer for insightful comments; and to INET for research support. tion, where the mapping between objective characteristics and subjective perceptions has been studied much more thoroughly. I then conjecture that the same kind of information-processing constraint may be relevant for perceptions of economic value.

Let us consider in particular the relation between the objective luminance of an object in one's field of vision (i.e., the amount of light actually reflected to the eye from it) and the subjective perception of its brightness. Experiments in psychophysics and in neurobiology have established a number of key points. ${ }^{1}$ (1) The ability to discriminate between stimuli of differing brightness is both imprecise and probabilistic: as the difference in luminance between two stimuli increases, the probability of a correct assessment of which is brighter increases continuously. (2) Perceived brightness depends on the contrast between an object's luminance and the background level of luminance to which the eye has adapted, rather than on the object's absolute level of luminance. (3) Finer discriminations are possible among degrees of contrast in the range that occur most frequently in the environment to which the eye has adapted.

The theory proposed in Woodford (2011) would explain these features of vision as follows. Suppose that a visual system adapts to process perceptions of brightness in an environment in which the objective level of $\log$ luminance is distributed according to some continuous distribution. Let visual processing be described by conditional probabilities $p(r \mid x)$ of a subjective perception of brightness $r$ in the case of objective $\log$ luminance $x .^{2}$ The degree of distor-

\footnotetext{
${ }^{1}$ See, e.g., Paul W. Glimcher (2011), chap. 12; Eric R. Kandel, James H. Schwartz, and Thomas M. Jessel, 2000, chap. 21; and additional references discussed in Woodford (2011).

${ }^{2}$ For simplicity, I here consider only perception of the brightness of a single, isolated light source, to make the problem unidimensional.
} 
tion of subjective perceptions can be measured by the mean squared error (MSE) of the optimal estimate $\hat{x}(r)$ of log luminance based on the subjective perception $r$. (Both the optimal estimate and the calculation of MSE depend, of course, on the distribution of levels of luminance to which the system is adapted.)

The efficient processing hypothesis then proposes that the probabilities $p(r \mid x)$ are chosen so as to minimize $M S E$, subject to an upper bound on the capacity $C$ required for a communication channel to be able to transmit a signal about $x$ that suffices to allow subjective representations $r$ to be generated with the desired probabilities. The required capacity is defined using information theory (Thomas M. Cover and Joy A. Thomas 2006) as

$$
C=\max _{\pi} \mathrm{E}_{\pi}\left[\log \frac{p(r \mid x)}{p(r)}\right],
$$

where $\pi$ is a prior over the frequency of occurrence of objective states $x$ and $p(r)$ is the implied unconditional frequency distribution for subjective representations $r$. Note that $C$ depends only on the conditional probabilities $p(r \mid x)$, and can be defined under arbitrary assumptions about the nature of subjective representations.

In Woodford (2011), I discuss the optimal information structure in the case that $\log$ luminance in the environment is distributed according to some Gaussian distribution $N\left(\mu, \sigma^{2}\right)$. It is found to involve only a finite number of possible subjective perceptions $r$, even though there are a continuum of values for objective luminance. Each of these subjective perceptions $r$ occurs with positive probability for all objective states $x$; thus perception is imprecise and stochastic. The probability that a stimulus with $x=\mu+z \sigma$ will be perceived to be brighter than one with $x=\mu$ is a continuously increasing, sigmoid function of $z$, equal to 0.5 when $z=0$, as with experimental "psychometric functions."

The theory also predicts that for a given value of $C$, the probability of a given subjective perception of brightness $r$ is a function of the normalized level of log lumi- nance, $z \equiv(x-\mu) / \sigma$, that is invariant under changes in $\mu$ and $\sigma$. This implies that when one shifts to an environment with a different mean luminance, the levels of objective luminance that are considered to be "bright" or "dark" shift. In fact, perceived brightness should depend only on contrast $z$, rather than on $x$. And because only a finite number of distinct subjective perceptions are possible, it is efficient for subjective perceptions to vary substantially with changes in $z$ only for values of $z$ in an interval around zero; only frequently occurring contrasts should be discriminated very well.

\section{A Theory of Inattentive Valuation}

I now propose a similar theory of efficient perceptual distortions in the context of estimation of the values of options faced by an economic decisionmaker (DM). Let each option be characterized by a value $x_{a}$ for each of several distinct attributes, indexed by $a$, and suppose that the true utility value of option $x$ is given by $u=$ $\sum_{a} x_{a}$. Suppose furthermore that each attribute must be perceived separately: there is a subjective perception $r_{a}$ for each attribute $a$, occurring with conditional probabilities $p_{a}\left(r_{a} \mid x_{a}\right)$, conditionally independent across attributes. My hypothesis is then that these conditional distributions minimize the MSE of the optimal subjective estimate $\hat{u}$ based on the vector of subjective representations, subject to an upper bound on the total required processing capacity $C \equiv \sum_{a} C_{a}$.

Let us consider the solution to this problem in the case that the prior distribution for the vector of attributes (with respect to which the perceptual system is optimized) is given by an independent Gaussian distribution $N\left(\mu_{a}, \sigma_{a}^{2}\right)$ for each attribute. Then for each attribute $a$, the probabilities $p_{a}\left(r_{a} \mid x_{a}\right)$ solve exactly the same mathematical problem as in the discussion of vision, for some capacity constraint $C_{a}$. In addition, the capacity $C_{a}$ allocated to the perception of each attribute is chosen so as to imply a Lagrange multiplier $\theta$ on the capacity constraint that is the same for each attribute. The optimal $C_{a}$ for any attribute 
can be shown to be a monotonically increasing function of $\sigma_{a}^{2} / \theta$, where the value of $\theta$ depends (inversely) upon the available total capacity $C$.

This implies that perceptions of value will be intrinsically stochastic, but that the degree of noise in subjective perceptions (relative to the range of variation in the true values) will be less in the case of attributes with larger values of $\sigma_{a}$, which is to say, attributes responsible for a larger share of the variation in true utility $u$ under the prior. Hence some attributes will be perceived more accurately than others; for a low enough share of the variation $\left(\sigma_{a}^{2}<\right.$ $0.75 \theta$ ), it is efficient to completely ignore the attribute in question.

For any given capacity $C_{a}>0$, the (finite) number of distinct gradations of perceived value that will be discriminated is independent of the parameters of the prior distribution, and the probability of any given subjective perception $r_{a}$ will depend only on the normalized value of the attribute, $z_{a} \equiv\left(x_{a}-\mu_{a}\right) / \sigma_{a}$, rather than on the absolute value $x_{a}$ of that attribute. A given subjective perception $r_{a}$ will correspondingly imply a particular estimate $\hat{z}_{a}$ of the normalized value; the option chosen should then be the one with the highest value of $\sum_{a} \sigma_{a} \hat{z}_{a}\left(r_{a}\right)$. Moreover, because it is efficient for subjective perceptions to vary substantially with changes in $z_{a}$ only for values of $z_{a}$ in an interval around zero, the mean normalized subjective value $\mathrm{E}\left[\hat{z}_{a}\right]$ will be a sigmoid function of the true normalized value $z_{a}$, with a slope that is greatest at $z_{a}=0$, as shown in Figure 1 below.

Such a theory can account for a number of seemingly anomalous features of observed choice behavior (Woodford 2011). Because the subjective perception of value is stochastic, choice is predicted to be intrinsically stochastic, as has long been observed in experiments, even in the absence of any random variations in utility (of the kind used, for example, by Daniel McFadden 1974 to explain stochastic choice).

The theory can account for "focusing illusions," in which choice gives disproportionate weight to a few particularly important features of the available options, while discounting (or wholly ignoring) a large number of other features, each individually of minor significance for utility, but that would cumulatively matter a great deal if more accurately weighed (Botond Koszegi and Adam Szeidl 2011). And it can account for the existence of context effects, such as "decoy effects," in which addition of another good to the choice set can increase demand for one of the previously available goods (Timothy B. Heath and Subimal Chatterjee 1995), if the distribution of attribute values to which the perceptual system is adapted is determined by the distribution of attribute values over the options in the choice set. Here, however, I focus on the explanation that the theory provides for reference-dependent valuations of economic choices.

\section{Reference-Dependent Choice}

The model just sketched implies that subjective valuations will depend not only on goods' objective attributes, but also on where the objective value falls within the distribution of anticipated possibilities. Thus perceptions of value are relative, leading to "reference-dependence" of the kind stressed by Kahneman and Tversky (1979).

For example, Kahneman and Tversky present different groups of experimental subjects with the following two choices:

- Problem 1: In addition to whatever you own, you have been given 1000 . You are now asked to choose between (a) winning an additional 500 with certainty, or (b) a gamble with a 50 percent chance of winning 1000 and a 50 percent chance of winning nothing.

- Problem 2: In addition to whatever you own, you have been given 2000 . You are now asked to choose between (a) losing 500 with certainty, and (b) a gamble with a 50 percent chance of losing 1000 and a 50 percent chance of losing nothing.

They report that substantial majorities of their subjects choose the sure thing in Problem 1, and the gamble in Problem 2. Yet 
in each of the two problems, the choice is between identical probability distributions over possible final wealth states; thus the evaluation of these options is evidently not merely a function of the probabilities assigned to different final wealth states. Kahneman and Tversky propose instead that in each problem, the different possible final wealths are evaluated relative to a " reference point" corresponding to the wealth possessed prior to the decision; it is the fact that the reference point is higher by 1000 in Problem 2 that results in a different evaluation of the relative attractiveness of the two lotteries.

The theory of inattentive valuation proposed here provides an explanation for such findings, that remains a variant of rational choice theory, and that still includes the standard (von Neumann-Morgenstern) theory of choice over lotteries as a limiting case (the case in which the processing capacity allocated to the evaluation of one's options is large enough). Suppose that we treat each of the possible outcomes for a lottery as separate attributes that must be evaluated. In the above example, we may suppose that each of option has two attributes: $(1500,1500)$ for option (a) and $(1000,2000)$ for option (b).

According to the theory presented above, the conditional distribution $p\left(r_{s} \mid x_{s}\right)$ over subjective representations in the case of a given objective final wealth $x_{s}$ in state $s$ is adapted to a particular distribution of possible final wealths associated with choice situations of that kind. The distribution to which the DM's perceptions of value are adapted may well be different in situations like Problem 1 than in situations like Problem 2 ; hence the subjective perceptions of options (a) and (b) may be different in the two problems, even though the probability distributions over final wealth in the two options are the same.

For example, suppose that the DM recognizes the class of choice situations in which "you have been given 1000, and now are asked to choose between lotteries" as different from the class of situations in which "you have been given 2000, and now are asked to choose between lotteries," and so perceives the options presented using a perceptual system that has been optimally adapted to different distributions of potential outcomes in the two cases. If the DM has no reason to expect the types of gains and losses that may be offered by the lotteries to depend on her wealth at the time that the choice is presented, then the prior distribution over possible levels of final wealth should indeed be different between the two classes of situations: the entire probability distribution should be shifted up by 1000 in the case of the second class.

For example, if the distribution of possible net gains from a lottery payoff in either state of the world is assumed to be a Gaussian distribution $N\left(0,1000^{2}\right)$, then for either state of the world, in both classes of choice situations the prior distribution over possible final wealths should be a Gaussian distribution $N\left(\mu, 1000^{2}\right)$, but $\mu$ should be higher by 1000 for the second class. Since the standard deviation is the same for both classes of situations, if they are also expected to occur with equal frequency (so that the DM cares equally about accurate evaluations of options in the two cases), it will be optimal for the same processing capacity $C$ to be allocated to perceptions of the value of outcomes in each of the two cases. Then if the optimal information structure for situations in class 1 involves conditional probabilities $\left\{p^{1}\left(r_{s} \mid x_{s}\right)\right\}$, the optimal information structure for situations in class 2 will involve conditional probabilities $\left\{p^{2}\left(r_{s} \mid x_{s}\right)\right\}$ defined over the same domain, where

$$
p^{2}\left(r_{s} \mid x_{s}\right)=p^{1}\left(r_{s} \mid x_{s}-1000\right)
$$

for each subjective perception $r_{s}$ and each possible final wealth level $x_{s}$.

Reversals of the preference ordering of the kind reported by Kahneman and Tversky can easily be explained by referencedependence of this sort in the way that subjective perceptions of value are coded. As an example, Figure 1 shows the mean normalized subjective value (MNSV) assigned to each lottery in each of the two choice situations just discussed, if the processing capacity allocated to the perception of the 


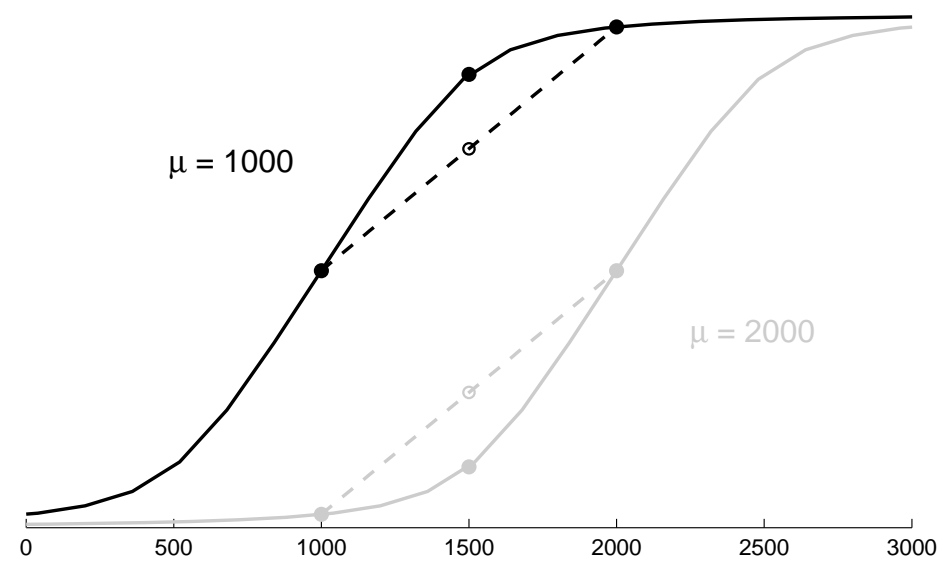

Figure 1. MEAN NORMalized SubJeCtive VAlue For LOtTeries in tWo CHOICE PROBlems.

Key: Solid dots: MNSV of a single outcome; open dots: MNSV of a lottery. Black line: initially given 1000; grey line: initially given 2000 .

value of outcomes in each class of situations is one-half a binary digit. Here the horizontal axis $x$ indicates the amount by which the DM's final wealth exceeds initial wealth, and the vertical axis plots $\hat{z}$, the DM's estimate of the normalized value. Each of the two sigmoid curves plots the function $\mathrm{E}[\hat{z} \mid x]$ for one of the two classes of choice situations: the black curve for class 1 (the prior with $\mu=1000$ ) and the grey curve for class 2 (the prior with $\mu=2000$ ). The lottery with the higher MNSV in any choice situation is the one that should on average be preferred (though actual choice will be noisy, owing to the stochasticity of subjective perception).

In the case of option (a), which results in $x=1500$ with certainty, the MNSV is given by the solid dot above $x=1500$ on the curve corresponding to the given choice situation. In the case of option (b), the MNSV for one state will be given by the solid dot above $x=1000$ on the appropriate curve, while the MNSV for the other state will be given by the black dot above $x=2000$ on that same curve. The overall MNSV for option (b), averaging the MNSVs for the two equiprobable states, will then be given by the open dot above $x=1500$, the midpoint of the dashed line connecting the two black dots representing the MNSVs for the individual states. The figure clearly shows that in this numerical example, option (a) should be preferred on average to option (b) in Problem 1 (the solid dot is higher than the open dot), while option (b) should be preferred on average to option (a) in Problem 2 (the open dot is higher than the solid dot, in this case). Hence the experimental results of Kahneman and Tversky are quite consistent with this model of valuation.

The theory predicts not only referencedependent valuations, but also the coexistence of apparent risk-aversion in the domain of gains with apparently risk-seeking behavior in the domain of losses. This is because efficient coding of perceptions of value involves diminishing sensitivity to further changes in value that are either well above or well below the levels that are expected to be encountered most often. The formal structure of the theory is similar to that of prospect theory (Kahneman and Tversky 1979), but a theoretical derivation is given here for the shape of the nonlinear "value function" postulated there. The present theory also clarifies that the "reference point" ( $\mu$ above) should in general be determined not by the status quo, but by the mean outcome that is expected in a given class of situations. 


\section{Conclusion}

Reference-dependent choice of the kind captured by prospect theory may be understood, then, as an efficient approach to choice by a DM with limited informationprocessing capacity. The theory provides an explanation for why biases of this kind in choice behavior should be so commonly observed, especially in experimental settings where the stakes for subjects may not be large enough to justify paying close attention. At the same time, it predicts that choices should conform more closely to standard theory in situations where the allocation of more processing capacity to the evaluation of options yields a sufficient benefit.

An important topic for further analysis is the determination of the prior distribution with respect to which the DM's perceptual system should be optimized in a given situation. In the example above, if situations in class 1 and class 2 were not distinguished (with a different expected distribution of possible outcomes for each class), there could be no difference in the "reference point" for the two cases. At the same time, if the DM's perceptual system were adapted to a different distribution for each of the options "(a) in situation 1," "(b) in situation 1," and so on, then - because the distributions of possible outcomes for "(a) in situation 1" and "(a) in situation 2 " are the same - there would again be no difference in the MNSV for option (a) in situation 1 as opposed to situation 2, and similarly for option (b).

Hence the kind of reference-dependence observed by Kahneman and Tversky (1979) can only be explained under the hypothesis that different levels of wealth achieved prior to the choice trigger the use of different perceptual codes, while the evaluation of different options within a given "choice situation" does not. ${ }^{3}$ Presumably the fact that the method of perceptual coding takes

\footnotetext{
${ }^{3} \mathrm{~A}$ similar comment applies to the theory of endogenous determination of the reference point proposed by Koszegi and Matthew Rabin (2006), which also identifies the reference point with the expected outcome in a given situation.
}

account of some, but not all, aspects of the DM's situation should itself be explained as a way of economizing on informationprocessing capacity; but such an inquiry is left for future work.

\section{REFERENCES}

Cover, Thomas M., and Joy A. Thomas. 2006. Elements of Information Theory. New York: Wiley, 2d ed.

Glimcher, Paul W. 2011. Foundations of Neuroeconomic Analysis. Oxford: Oxford University Press.

Heath, Timothy B., and Subimal Chatterjee. 1995. "Asymmetric Decoy Effects on Lower-Quality versus HigherQuality Brands: Meta-Analytic and Experimental Evidence." Journal of Consumer Research, 22: 268-284.

Kahneman, Daniel, and Amos Tversky. 1979. "Prospect Theory: An Analysis of Decision Under Risk." Econometrica, 47: 263-291.

Kandel, Eric R., James H. Schwartz, and Thomas M. Jessell. 2000. Principles of Neural Science. New York: McGraw-Hill, 4th ed.

Koszegi, Botond, and Matthew Rabin. 2006. "A Model of ReferenceDependent Preferences." Quarterly Journal of Economics, 121: 1133-1165.

Koszegi, Botond, and Adam Szeidl. 2011. "A Model of Focusing and Economic Choice." Unpublished.

McFadden, Daniel. 1974. "Conditional Logit Analysis of Qualitative Choice Behavior." In Frontiers in Econometrics, ed. Paul Zarembka, 105142. New York: Academic Press. Sims, Christopher A. 2003. "Implications of Rational Inattention." Journal of Monetary Economics, 50: 665690.

Woodford, Michael. 2011. "Inattentive Valuation and ReferenceDependent Choice." Unpublished. 